\title{
Future developments in left ventricular assist device therapy
}

\author{
Andrew Shaffer, MD, ${ }^{\mathrm{a}}$ Rebecca Cogswell, MD, ${ }^{\mathrm{b}}$ and Ranjit John, $\mathrm{MD}^{\mathrm{a}}$
}

Feature Editor's Introduction-Durable left ventricular assist device (LVAD) therapy has had a remarkable evolution over the past 2 decades. Moving from a therapy once confined to only bridge to transplant application with large pulsatile pumps, LVAD therapy now experiences widespread dissemination and adoption for patients with advanced heart failure, irrespective of heart transplant eligibility, utilizing miniature continuous flow centrifugal pumps with total magnetic levitation. Technological enhancements in pump design has been the major driving force for improved patient outcomes, but refinement of surgical techniques and patient management have made substantial contributions to current patient longevity on LVAD support. The near elimination of the risk for pump thrombosis and important reductions in risk of stroke with newer technology have paved the way for consideration of LVAD therapy for patients with less-advanced stages of heart failure. Despite this tremendous progress, a significant burden of adverse events remain that include stroke, nonsurgical bleeding, and infection that are associated with intense medical provider resource utilization and hospital readmissions that hamper further adoption. In this issue of the Journal, Shaffer and colleagues provide a valuable overview of the future developments in the field of durable LVAD therapy. Shaffer and colleagues review new strategies to address the hurdles associated with LVAD therapy, including improvements in surgical techniques, patient management, and novel device technology. Minimally invasive surgical approaches to pump implantation, clinical trials investigating aspirin-sparing medical management protocols and other anticoagulation strategies, and totally implantable pump technology are just a few of the important developments that will likely have important influences on the field in the near future. With numerous continuing developments in the field, durable

From the ${ }^{\mathrm{a} D i v i s i o n}$ of Cardiothoracic Surgery, Department of Surgery, and ${ }^{\mathrm{b}}$ Division of Cardiology, Department of Medicine, University of Minnesota, Minneapolis, Minn.

Received for publication April 7, 2020; revisions received July 17, 2020; accepted for publication July 25, 2020; available ahead of print Sept 16, 2020.

Address for reprints: Andrew Shaffer, MD, Division of Cardiothoracic Surgery, Department of Surgery, University of Minnesota, Minneapolis, MN 55455 (E-mail: shaff163@umn.edu).

J Thorac Cardiovasc Surg 2021;162:605-11 $0022-5223 / \$ 36.00$

Copyright (c) 2020 Published by Elsevier Inc. on behalf of The American Association for Thoracic Surgery

https://doi.org/10.1016/j.jtcvs.2020.07.125

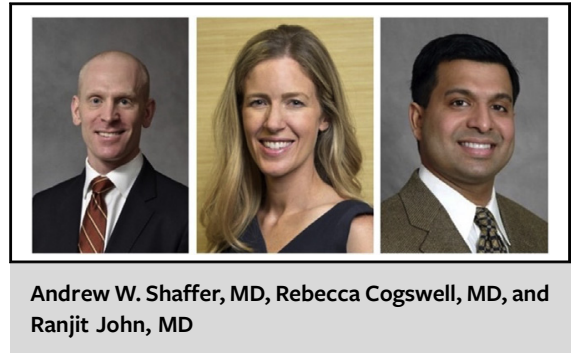

\begin{abstract}
CENTRAL MESSAGE
LVADs have dramatically

improved survival for patients with end-stage heart failure over

the past 2 decades. Although

adverse event rates are lower with contemporary LVADs, these events continue and limit wider adoption of device therapy. In this article, we review the current state of LVAD therapy and focus on future targets of device therapy advancement.
\end{abstract}

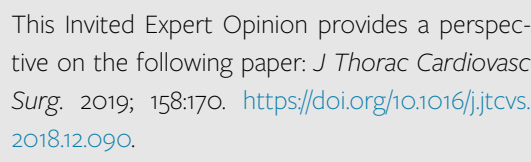

See Commentaries on pages 612,613 , and 614

LVAD therapy is poised to make ongoing challenges to heart transplantation.

\section{Francis D. Pagani, MD, PhD}

Significant advancements in the field of durable left ventricular assist devices (LVADs) have taken place during the past 2 decades, leading to improvements in morbidity and mortality after device implantation. ${ }^{1-5}$ Despite these advancements, hemocompatibility-related adverse events (eg, bleeding and thrombosis), stroke, device-related infections, and right ventricular failure continue to contribute to morbidity and mortality. A patient with an ideal LVAD would not 
require anticoagulation therapy, would have no associated thrombosis or bleeding events, and the device would be placed minimally invasively, be fully implantable, and would have a durability and efficacy that rival heart transplantation. All of these goals may not be achievable, but they highlight where the field is trying to evolve. In this article, we will review the current state of LVADs as well as future targets for advancement.

\section{CURRENT STATE OF DURABLE LVAD THERAPY}

Three main continuous flow pumps have made up the majority of LVADs implanted in the United States and Europe over the past 10 years. These include the Heart Mate II (HM2), the HeartMate III (HM3) (both from Abbott Medical, Chicago, Ill) and the Heartware HVAD (Medtronic, Minneapolis, Minn) (Figure 1). The HM3 now makes up the majority of implants in the United States as demonstrated in the most recent Interagency Registry for Mechanically Assisted Circulatory Support (INTERMACS) report. $^{9}$

\section{Pump Designs}

The axial flow HM2, which demonstrated superiority over the HeartMate I XVE (Thoratec, Pleasanton, Calif) pulsatile pump in $2008,{ }^{3}$ has now largely been replaced by pumps with magnetically levitated centrifugal designs (eg, HVAD and HM3). Between the HVAD and the HM3, the HVAD has smaller componentry (eg, pump body, outflow graft, and driveline). The smaller size of the HVAD is advantageous in applications for patients with smaller stature, minimally invasive approaches, and in the rare need for biventricular support. However, HM3 has also been utilized in all of these clinical scenarios. The smaller size of the centrifugal pumps overall allows intrapericardial implantation. Redo surgery for these devices is marginally less complicated due to the reduced extent of dissection of the preperitoneal space, left pleural space, and rarely intraperitoneal space.

\section{Summary of Primary End Points of Contemporary Multicenter LVAD Clinical Trials}

The HeartWare Ventricular Assist System as Destination Therapy of Advanced Heart Failure (ENDURANCE) trial was a randomized controlled trial of HVAD versus HM2 in a destination therapy (DT) population. The primary end point of the study was composite survival free from disabling stroke or need for device exchange at 2 years. Therapy was deemed equivalent with regard to the composite outcome $(55.4 \%$ for HVAD vs $59.1 \%$ for HM2). The rates of stroke and transient ischemic attack (TIA) were significantly higher in the HVAD group (0.29 events per patient-year of support vs 0.09 events per patient-year of support for HM2). ${ }^{4}$

The Multicenter Study of Maglev Technology in Patients Undergoing Mechanical Circulatory Support Therapy with HeartMate 3 (MOMENTUM 3) trial ${ }^{6,7}$ was a randomized trial of the HM3 centrifugal-flow LVAD against the HM2 LVAD. The study included 1028 patients with advanced-stage heart failure and included both bridge to transplantation (BTT) or as DT populations. ${ }^{6,7}$ The 2-year data were published in 2019 and the HM3 was found to be superior to the HM2 with respect to survival free of disabling stroke or reoperation to replace or remove a malfunctioning device. There has yet to be a direct comparison between the HVAD and the HM3. Comparison between the pumps can be inferred from the ENDUR$\mathrm{ANCE}^{4}$ and MOMENTUM 3 trials $^{6,7}$; however, it is important to note that trial participants were different

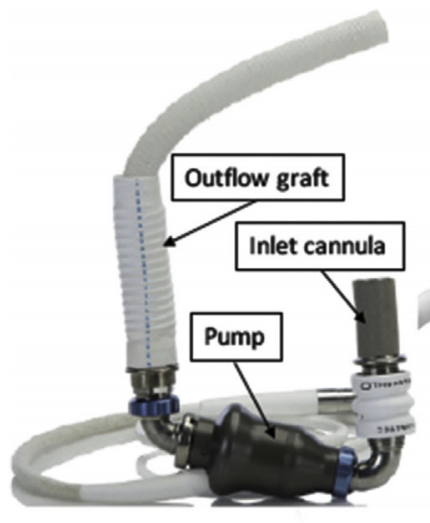

HeartMate II

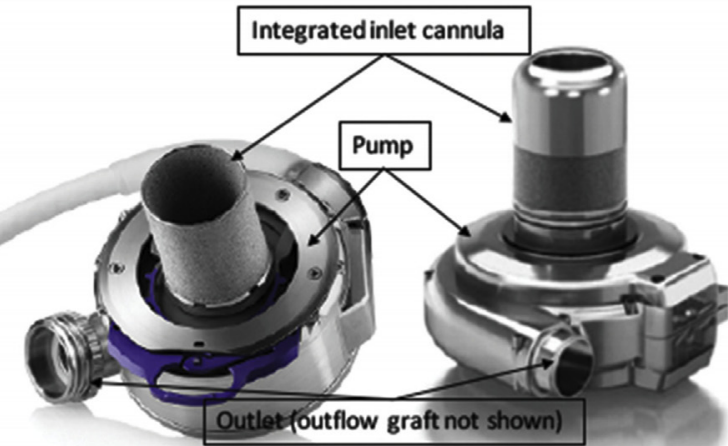

HVAD

FIGURE 1. Contemporary continuous flow left ventricular assist devices. Reprinted with permission from Stulak JM, El Ela AA, Pagani FD. Implantation of a durable left ventricular assist device: how I each it. Ann Thorac Surg. 2017;103:1687-92. HVAD, HeartWare left ventricular assist device. 
(ENDURANCE was DT only) as were the definitions of neurologic events.

\section{FUTURE DIRECTIONS TO REDUCE SPECIFIC ADVERSE EVENTS}

Adverse events, including all cause stroke, driveline infection, right heart failure, and gastrointestinal bleeding remain high on LVAD support even in contemporary clinical trials and registries. These events negatively influence quality of life and survival, and limit wider adoption of LVAD therapy to the growing population of end-stage heart failure patients. In this section, we will review the rates of adverse events observed in clinical trials and outline the steps the field is taking to try to reduce these events.

In the MOMENTUM 3 trial, the patients supported with the HM3 had lower rates of ischemic or hemorrhagic strokes of any severity and less bleeding events compared with patients supported by the HM2 over 2 years of support. The percentage of patients who developed TIA and right heart failure was similar between pumps. A summary of adverse events by pump type in MOMENTUM 3 and ENDURANCE trials is presented in Table 1. With respect to driveline infection rates, the ENDURANCE trial demonstrated an incidence of driveline infection of $16.2 \%$ for the HVAD and $12.1 \%$ for the HM2 over 2 years. ${ }^{4,5}$ The 2 -years data for MOMENTUM 3 demonstrated an incidence of driveline infection of $23 \%$ and $19 \%$ for the HM3 and HM2, respectively. ${ }^{6,7}$ These data suggest there is opportunity for improvement to LVAD technology to decrease device-related infection. Ultimately, elimination of the percutaneous driveline via total implantable technology may improve infectious complications. This will need to be proven clinically.

The ENDURANCE Supplemental Trial was designed to have more aggressive clinical management given the association between higher mean arterial blood pressure and stroke in patients supported with HVAD in the original ENDURANCE trial. The observed stroke rates in HVAD recipients were lower in the Supplement Trial. The primary end point was 12-month freedom from TIA or stroke with no residual deficit 24 weeks postevent. The primary end point was reached in $14.7 \%$ of patients in the HVAD arm and $12.1 \%$ in those supported with a HM2. ${ }^{5}$

\section{FUTURE DIRECTIONS WITH HEMOCOMPATIBILITY}

The HM3 was found to be a superior pump to the HM2 with respect to all clotting and bleeding events, although hemocompatibility-related adverse events continue to occur. A crucial goal for the community is to address anticoagulation and antiplatelet strategies in the HM3 population to decrease bleeding without increasing thrombotic events. The magnetically levitated centrifugal pump does not degrade high-molecular-weight multimers of Von Willebrand factor to the extent observed with other devices. ${ }^{5}$ The Antiplatelet Removal and HemocompatIbility Events With the HeartMate 3 Pump IDE Study (ARIES) trial is now underway in the United States, a randomization between acetylsalicylic acid $100 \mathrm{mg}$ and placebo and with standard international normalized ratio targets in new HM3 implants. ${ }^{6}$ The Minimal Anti-Coagulation Evaluation to Augment Hemocompatibility was previously published. That pilot study assessed an international normalized ratio

TABLE 1. Outcomes and characteristics of large, contemporary left ventricular assist device clinical trials*

\begin{tabular}{|c|c|c|c|c|}
\hline \multirow[b]{2}{*}{ Variable } & \multicolumn{2}{|c|}{ MOMENTUM 3 (N = 1028) } & \multicolumn{2}{|c|}{ ENDURANCE $(\mathbf{N}=\mathbf{4 4 5})$} \\
\hline & HM3 & HM2 & HVAD & HM2 \\
\hline \multicolumn{5}{|l|}{ Study characteristic/outcome } \\
\hline Cohort size by device & 516 & 512 & 296 & 149 \\
\hline Population included & \multicolumn{2}{|c|}{ DT/BTT/BTR } & \multicolumn{2}{|c|}{ DT } \\
\hline Length of follow-up (y) & \multicolumn{2}{|c|}{2} & \multicolumn{2}{|c|}{2} \\
\hline Successful primary end point & $397(76.9)$ & $332(64.8)$ & $164(55.2)$ & $85(57.4)$ \\
\hline 2-y survival $(\%)$ & 79 & 77 & 65 & 73 \\
\hline \multicolumn{5}{|l|}{ Adverse events } \\
\hline Exchange due to pump thrombosis & $5(1.0)$ & $56(11.1)$ & $19(6.4)$ & $16(10.7)$ \\
\hline All-cause stroke & $51(9.9)$ & $98(19.4)$ & $88(29.7)$ & $18(12.1)$ \\
\hline Transient ischemic attack & $16(3.1)$ & $19(3.8)$ & $25(8.4)$ & $7(4.7)$ \\
\hline Driveline infection & $120(23.3)$ & $98(19.4)$ & $58(19.6)$ & $23(15.4)$ \\
\hline Right heart failure & $176(34.2)$ & $143(28.3)$ & $114(38.5)$ & $40(26.8)$ \\
\hline Gastrointestinal bleeding & $126(24.5)$ & $156(30.9)$ & $104(35.1)$ & $51(34.2)$ \\
\hline
\end{tabular}

Values are presented as n (\%) unless otherwise noted. $H M 3$, HeartMate3; HM2, HeartMate2; HVAD, HeartWare left ventricular assist device; $D T$, destination therapy; $B T T$, bridge to transplant; $B T R$, bridge to recovery. *ENDURANCE and MOMENTUM primary end points: 2 years free of disabling stroke or reoperation to replace or remove a malfunctioning device. 
target range of 1.5 to 1.9 with acetylsalicylic acid $81 \mathrm{mg}$ daily in a small group of HM3 recipients. ${ }^{7}$ These data had a favorable safety profile, setting the stage for a larger randomized controlled trial.

\section{MOVING AWAY FROM THE TRADITIONAL BTT AND DT DESIGNATIONS}

For the past 2 decades, patients receiving LVADs were broadly categorized into 2 groups: BTT and DT. Although early on these groups were clinically dissimilar, later the 2 populations demonstrated overlap. Patients, who were previously deemed unsuitable transplant candidates and offered an LVAD as DT or as bridge to decision, subsequently become candidates for transplantation when the relative contraindications for transplantation no longer exists (eg, pulmonary hypertension, compliance, and obesity). In some situations, LVAD candidates become acceptable candidates for transplant when they develop 1 or more possible LVAD complications because the only definite cure remains heart transplantation with LVAD explantation. Slaughter and colleagues ${ }^{3}$ demonstrated in the 2008 HeartMate II trial that $17 \%$ of DT LVAD patients underwent transplant by 2 -year follow-up. ${ }^{3}$ This, as well as other observation of frequent designation changes, contributed to the change in trial format for the MOMENTUM study, which instead used short- and long-term designations. ${ }^{4,5}$ The same inclusion criteria were used for both categories of patients. The absolute benefit of the HM3 pump was not altered by the intended goal of therapy. In the MOMENTUM study, $15 \%$ of those initially deemed transplant ineligible were eventually transplanted within 2 years of follow-up. ${ }^{8}$ It is likely that future clinical trials in durable mechanical support will use the same short- and longterm designations. Reimbursement and regulatory requirements may also follow this change in designation.

\section{Effect of United Network for Organ Sharing Allocation on Current and Future LVAD Use}

The heart allocation system was redesigned in the United States October 2018. The major change to the new system was separation of the previous high urgency status (1A) into 3 separately ranked statuses (new status 1,2, and 3). Early analyses of the system demonstrate that temporary support has increased substantially and that fewer LVAD patients are receiving transplants. ${ }^{9-11}$ In addition, there has been a substantial shift in percent of LVADs that are placed as BTT as reported by the 2019 INTERMACS report. ${ }^{12}$ Although the donor pool may eventually be expanded with hepatitis $\mathrm{C}$ donors and potentially donation after cardiac death, this will not be enough, given the rising prevalence of heart failure and increased survival of suitable candidates on LVAD support. If more programs adopt a temporary support strategy, wait times for the highest acuity statuses will necessarily lengthen. This may shift practice patterns toward placing LVADs again as BTT. As patients with LVADs who are listed for transplant now will have a longer wait time before transplant, LVAD durability will be tested.

\section{Totally Implantable LVADs}

A totally implantable LVAD system represents the next logical advancement in LVAD design, given the potential for reduced infections and improvement in quality of life. Thermal energy transfer system (TETS) technology has been incorporated into numerous mechanical support designs, but only 2 have been used clinically: the AbioCor total artificial heart (Abiomed, Danvers, Mass) and the LionHeart LVAD (Arrow International, Reading, Pa). Neither the AbioCor nor the LionHeart studies achieved acceptable long-term survival; however, the incidence of device-related infections was lower than devices with external components. In both studies, the TETS was reliable for at least 1 to 2 years. ${ }^{13-15}$

For totally implantable technology to be practical, several features are needed. First, a long-lasting and rapidly chargeable implantable battery. Second, the batteries and controllers should be small to minimize complications from abdominal or thoracic wall placement. Third, the design should not damage the skin and surrounding tissues and should accommodate a wide range of patient sizes and allow for a variety of patient activities.

Several in vitro and animal studies have suggested possible solutions, including use of a coplanar energy transfer (CET) system. ${ }^{16}$ Unlike TETS, the unique architecture of the CET system is characterized by 2 large rings utilizing coil-within-the-coil topology, ensuring high and robust resonance energy powering. Pya and colleagues ${ }^{17}$ recently described 2 cases of a CET technology that ameliorates infection risk by driveline elimination while providing successful energy transmission and allowing for substantial (8.5 hours) unholstered support while on the LVAD. The unique system architecture meets maximum allowable temperature deviation of implanted components, as consistently validated in long-term animal studies. ${ }^{17}$ It is likely that the device manufactures will focus on the development of totally implantable systems in the coming years.

\section{Surgical Advances}

Minimally invasive LVAD surgery has been made possible by improvements in device performance and outcomes combined with miniaturization in device size. ${ }^{18-20}$ The newer surgical techniques focus on 2 main areas: less-invasive cardiac access and off-pump technique. LVAD implantation via a thoracotomy approach has several 
potential advantages: reduction in adhesions for later reoperations, reduction of surgical trauma and peri- and postoperative bleeding, reduction in sternal wound complications, cosmetic benefits, and potential reduction in postoperative right ventricular failure. Preserving the pericardium using lateral thoracotomy approach maintains right ventricular geometry and pressure-volume relationship. In addition, acute right ventricular compression that occurs in the sternotomy approach is avoided. Finally, less transfusion of blood products with this approach may be protective to the lungs and right ventricle. The Evaluation of a Lateral Thoracotomy Implant Approach for a Centrifugal-Flow Left Ventricular Assist Device: The LATERAL Clinical Trial (LATERAL) was a multicenter, prospective, and historical control study in select BTT patients to evaluate the safety and effectiveness of HVAD implantation via a thoracotomy approach. Results demonstrated an acceptable safety profile with adverse event rates comparable to previous HVAD studies. ${ }^{18}$

The benefit of off-pump surgery is the potential reduction of systemic inflammatory response induced by cardiopulmonary bypass. Fibrinolysis, platelet sequestration, and degradation of coagulation factors have all been documented. LVAD implantation is also associated with high rates of postoperative vasoplegia. LVAD implantation without cardiopulmonary bypass could potentially reduce the burden of these complications. With currently available technology, the use of the off-pump technique is relatively limited.

Patients who may not be appropriate for minimally invasive or off-pump thoracotomy approach include those who require concomitant cardiac valve procedures or those with significant left-ventricular thrombus. Technical challenges with this technique can also lead to malposition or unrecognized cardiac injuries. Whether off-pump or minithoracotomy or a combination of both will be the predominant implanting technique in the future remains to be determined.

\section{Mini-LVAD}

The concept of a miniature LVAD is not new. A support device that is smaller and that could be placed less invasively has a number of potential applications. An intermediate amount of hemodynamic support may be enough for some patients to bridge to candidacy, transplant, or recovery. Such a device could also support different types of physiology (restrictive or smaller left ventricle cavity size) and may provide enough support for patients with biventricular failure as a bridge without the risk of right ventricle decompensation that can occur with LVAD implantation. This was the concept with CircuLite SYNERGY (CircuLite, Saddle Brook, NJ), which faced technical challenges and is the concept for NuPulse (NuPulseCV, Raleigh,

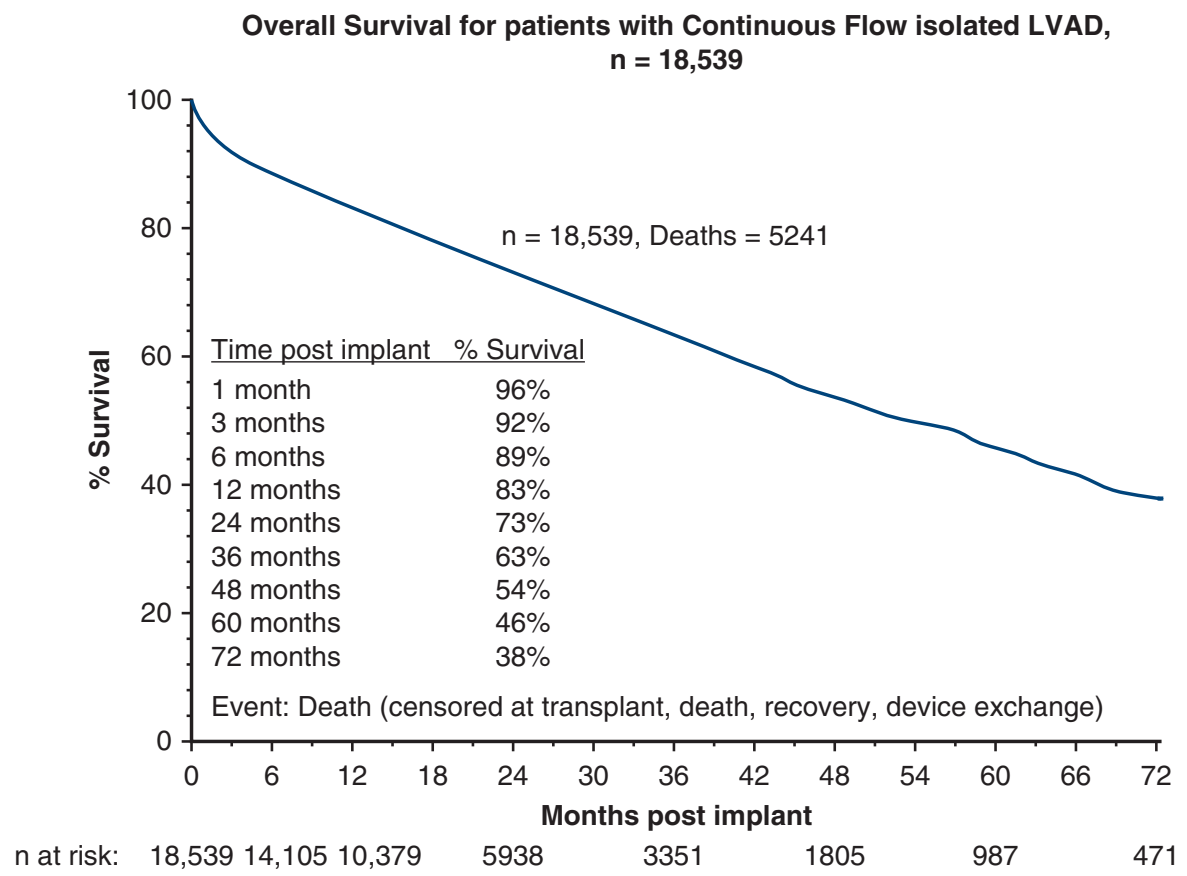

FIGURE 2. Overall survival for patients with continuous flow isolated LVADs. Reprinted with permission from Kormos RL, Cowger J, Pagani FD, Teuteberg JJ, Goldstein DJ, Jacobs JP, et al. The Society of Thoracic Surgeons intermacs Database annual report: evolving indications, outcomes, and scientific partnerships. Ann Thorac Surg. 2019;107:341-54. 
NC), which is presently in a feasibility trial across the United States. $^{21,22}$

\section{Improving Implantation Timing, and Increasing Appropriate Referrals}

Harnessing technological advances and artificial intelligence has several potential applications for the field of advanced heart failure: delivery of high-quality heart failure care to a broader population, increased referrals to advanced heart failure centers, and identification of optimal LVAD implantation timing.

Data that could be utilized for such algorithms include the electronic medical record (trend in renal function, B-type natriuretic peptide, and frequency of emergency room visits or hospitalizations), implantable monitors (CardioMEMS [Abbott Medical] and pacemaker/defibrillator data), patient-reported symptoms and potentially smartphone or apple watch data (eg, heart rate, activity level, self-administered 6-minute walk, or quality of life scores). ${ }^{23,24}$

As an example, a group from South Korea developed a deep-learning-based artificial intelligence algorithm for predicting the mortality of patients with acute decompensated heart failure using 2 hospital datasets (1 for development and 1 for validation). This model outperformed conventional heart failure mortality risk scores. ${ }^{25}$ Because there is great practice variability in timing of referral to advanced heart failure programs and known increase in risk of adverse outcomes related to late implantation, we believe there is large potential for more development in this area. Harnessing such technology has the ability to deliver care to a larger population.

\section{Comparisons of LVADs to Heart Transplantation}

Data from MOMENTUM 3, INTERMACS, ${ }^{9}$ and the International Heart Lung Transplant Society reports can be used to compare contemporary survival after LVAD implantation to cardiac transplantation. The survival for HM3 patients was $79 \%$ at 24 months from the time of LVAD implantation. ${ }^{6,7}$ In the 2019 INTERMACS report (spanning LVAD implants between 2008-2017), the survival after LVAD implantation was $73 \%$ at 24 months (Figure 2). ${ }^{9}$ Data from a 2017 International Heart Lung Transplant Society report show survival after heart transplant to be $82 \%$ at 24 months from surgery. ${ }^{10}$ These data demonstrate that survival after heart transplant remains superior to LVAD implantation at the 2-year mark. The survival of HM3 patients at the 3- and 5-year mark remains unknown. Because the donor shortage will remain and more suitable recipients are supported on LVADs, the ultimate goal in the field of mechanical support should be to deliver outcomes that are comparable to heart transplant.

\section{CONCLUSIONS}

This is a sobering reminder of the limited applicability of heart transplantation because the donor shortage has already shown us where the next frontier of clinical progress should be: Mechanical circulatory support. An increasing amount of evidence suggests that the use of arbitrary categorizations based on current or later transplant eligibility should be clinically abandoned in favor of a single preimplant strategy. This step alone could relieve many of the regulatory issues that sometimes limit clinical progress. Focus on further reduction of adverse events via several strategies and a steady stepwise development of a totally implantable LVAD will be the highlights in the field of LVAD in the foreseeable future.

\section{Conflict of Interest Statement}

Dr Cogswell is part of the Heart Failure Advisory Board for Medtronic, and the speaker's bureau for Abbott Medical. Dr John is the recipient of research grants and is a consultant for Abbott Medical and Medtronic. Dr Shaffer reported no conflicts of interest.

The Journal policy requires editors and reviewers to disclose conflicts of interest and to decline handling or reviewing manuscripts for which they may have a conflict of interest. The editors and reviewers of this article have no conflicts of interest.

\section{References}

1. Frazier OH, Rose EA, McCarthy P, Burton NA, Tector A, Levin H, et al. Improved mortality and rehabilitation of transplant candidates treated with a long-term implantable left ventricular assist system. Ann Surg. 1995;222:327-36.

2. Rose EA, Gelijns AC, Moskowitz AJ, Heitjan DF, Stevenson LW, Dembitsky W, et al; Randomized Evaluation of Mechanical Assistance for the Treatment of Congestive Heart Failure (REMATCH) Study Group. Long-term use of a left ventricular assist device for end-stage heart failure. $N$ Engl J Med. 2001;345: 1435-43.

3. Slaughter MS, Rogers JG, Milano CA, Russell SD, Conte JV, Feldman D, et al; HeartMate II Investigators. Advanced heart failure treated with continuous-flow left ventricular assist device. N Engl J Med. 2009;361:2241-51.

4. Mehra MR, Uriel N, Naka Y, Cleveland JC Jr, Yuzefpolskaya M, Salerno CT, et al. A fully magnetically levitated left ventricular assist device-the final cohort. N Engl J Med. 2019;380:1618-27.

5. Mehra MR, Naka Y, Uriel N, MOMENTUM 3 Investigators. A fully magnetically levitated circulatory pump for advanced heart failure. $N$ Engl $\mathrm{J}$ Med. 2017;376:440-50.

6. The ARIES HeartMate 3 Pump IDE Study. Available at: https://clinicaltrials.gov/ ct2/show/NCT04069156. Accessed September 19, 2020.

7. Netuka I, Ivák P, Tučanová Z, Gregor S, Szárszoi O, Sood P, et al. Evaluation of low-intensity anti-coagulation with a fully magnetically levitated centrifugalflow circulatory pump-the MAGENTUM 1 study. J Heart Lung Transplant. 2018;37:579-86.

8. Goldstein DJ, Naka Y, Horstmanshof D, Ravichandran AK, Schroder J, Ransom J, et al. Association of clinical outcomes with left ventricular assist device use by bridge to transplant or destination therapy intent: the multicenter study of MagLev technology in patients undergoing mechanical circulatory support therapy with HeartMate 3 (MOMENTUM 3) randomized clinical trial. JAMA Cardiol. 2020;5:411-9.

9. Stevenson LW, Kormos RL, Young JB, Kirklin JK, Hunt SA. Major advantages and critical challenge for the proposed United States heart allocation system. $J$ Heart Lung Transplant. 2016;35:547-9. 
10. Barr ML, Taylor DO. Changes in donor heart allocation in the United States without fundamental changes in the system: rearranging deck chairs and elephants in the room. Am J Transplant. 2015;15:7-9.

11. Cogswell R, John R, Estep JD, Duval S, Tedford RJ, Pagani FD, et al. An early investigation of outcomes with the new 2018 donor heart allocation system in the United States. J Heart Lung Transplant. 2020;39:1-4.

12. Teuteberg JJ, Cleveland JC Jr, Cowger J, Higgins RS, Goldstein DJ, Keebler M, et al. The Society of Thoracic Surgeons INTERMACS 2019 annual report: the changing landscape of devices and indications. Ann Thorac Surg. 2020;109: 649-60.

13. Slaughter MS, Myers TJ. Transcutaneous energy transmission for mechanical circulatory support systems: history, current status, and future prospects. $J$ Card Surg. 2010;25:484-9.

14. Mehta SM, Pae WE Jr, Rosenberg G, Snyder AJ, Weiss WJ, Lewis JP, et al. The LionHeart LVD-2000: a completely implanted left ventricular assist device for chronic circulatory support. Ann Thorac Surg. 2001;71(3 Suppl):S156-61.

15. Dowling RD, Gray LA Jr, Etoch SW, Laks H, Marelli D, Samuels L, et al. Initial experience with the AbioCor implantable replacement heart system. J Thorac Cardiovasc Surg. 2004;127:131-41.

16. Weiss WJ, Rosenberg G, Snyder AJ, Donachy J Sr, Reibson J, Kawaguchi O, et al. A completely implanted left ventricular assist device. Chronic in vivo testing. ASAIO J. 1993;39:M427-32.

17. Pya Y, Maly J, Bekbossynova M, Salov R, Schueler S, Meyns B, et al. First human use of a wireless coplanar energy transfer coupled with a continuous-flow left ventricular assist device. J Heart Lung Transplant. 2019; 38:339-43.

18. McGee E Jr, Danter M, Strueber M, Mahr C, Mokadam NA, Wieselthaler G, et al. Evaluation of a lateral thoracotomy implant approach for a centrifugal-flow left ventricular assist device: the LATERAL clinical trial. J Heart Lung Transplant. 2019;38:344-51.

19. Maltais S, Anwer LA, Tchantchaleishvili V, Haglund NA, Dunlay SM Aaronson KD, et al. Left lateral thoracotomy for centrifugal continuous-flow left ventricular assist device placement: an analysis from the mechanical circulatory support research network. ASAIO J. 2018;64:715-20.

20. Ricklefs M, Hanke JS, Dogan G, Napp LC, Feldmann C, Haverich A, et al. Less invasive surgical approaches for left ventricular assist device implantation. Semin Thorac Cardiovasc Surg. 2018;30:1-6.

21. Barbone A, Pini D, Rega F, Ornaghi D, Vitali E, Meyns B, et al. Circulatory support in elderly chronic heart failure patients using the CircuLite ${ }^{\circledR}$ Synergy ${ }^{\circledR}$ system. Eur J Cardiothorac Surg. 2013;44:207-12.

22. Jeevanandam V, Song T, Onsager D, Ota T, LaBuhn CJ, Lammy T, et al. The firstin-human experience with a minimally invasive, ambulatory, counterpulsation heart assist system for advanced congestive heart failure. J Heart Lung Transplant. 2018;37:1-6.

23. Gronda E, Vanoli E, Zorzi A, Corrado D. CardioMEMS, the real progress in heart failure home monitoring. Heart Fail Rev. 2020;25:93-8.

24. Barrett M, Boyne J, Brandts J, Brunner-La Rocca HP, De Maesschalck L, De Wit K, et al. Artificial intelligence supported patient self-care in chronic heart failure: a paradigm shift from reactive to predictive, preventive and personalised care. EPMA J. 2019;10:445-64.

25. Kwon JM, Kim KH, Jeon KH, Lee SE, Lee HY, Cho HJ, et al. Artificial intelligence algorithm for predicting mortality of patients with acute heart failure. PLoS One. 2019;14:e0219302.

Key Words: heart failure, ventricular assist device 